\title{
Is it possible to not perform salivary gland biopsy in targeted patients according to unstimulated salivary flow results in patients with suspected Sjögren's syndrome?
}

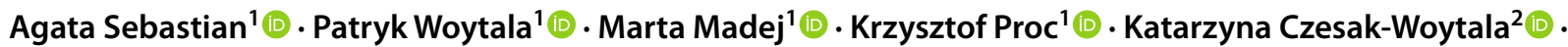 \\ Maciej Sebastian ${ }^{3}$. Krzysztof Zub ${ }^{4}$ iD $\cdot$ Piotr Wiland $^{1}$ (iD
}

Received: 30 January 2021 / Accepted: 8 March 2021 / Published online: 12 April 2021

(c) The Author(s) 2021

\begin{abstract}
Introduction/objective Xerostomia is one of the main symptoms of primary Sjögren's syndrome (pSS). The unstimulated salivary flow (UWS) test is one of the objective Sjögren's syndrome classification criteria used to assess xerostomia's severity. The study's objective was to evaluate UWS rate measurements (with a threshold rate of $0.1 \mathrm{~mL} / \mathrm{min}$ ) in the screening of patients suspected with pSS, presenting with xerostomia in whom labial salivary gland biopsy (LSGB) should be performed. We will try to answer whether it is possible not to perform LSGB in targeted patients according to UWS results? We analyze the correlation between UWS value and focus score (FS) and anti-SSA antibodies.

Methods The study group consisted of subjects above 18 years of age with a subjective feeling of oral dryness.

Results A total of 105 subjects were qualified for the study. The final diagnosis of pSS was made in 44 patients according to the classification criteria from 2016. No age differences were identified between pSS patients and control group subjects (patients with dry mouth without autoimmune background). UWS rates were significantly lower in pSS patients than in the control group. No association was identified between UWS and focus score (FS) $\geq 1$ in LSGB. No differences were observed between anti-SSA-positive and anti-SSA-negative patients in terms of age, UWS rates, FS.

Conclusion LSGB should be performed in all suspected pSS cases regardless of the UWS rate value, particularly in subjects without specific anti-SSA antibodies. In patients with suspected pSS, only less than one-half of the UWS measurements are below the value of $0.1 \mathrm{~mL} / \mathrm{min}$ adopted as the threshold in the classification criteria for pSS.
\end{abstract}

Keywords Primary Sjögren's syndrome $\cdot$ Hyposalivation $\cdot$ Unstimulated salivary flow $\cdot$ Focus score $\cdot$ Xerostomia

\section{Introduction}

Agata Sebastian

agatasebastian@vp.pl

1 Department of Rheumatology and Internal Medicine, Medical University of Wroclaw, Borowska 213, 50-556 Wroclaw, Poland

2 Department of Mathematics and Cybernetics, Wroclaw University of Economics, Wroclaw, Poland

3 Department of General, Minimally Invasive and Endocrine Surgery, Medical University of Wroclaw, Wroclaw, Poland

4 Department and Clinic of Otolaryngology-Head and Neck Surgery, Wroclaw Medical University, Wroclaw, Poland
Xerostomia is one of the main symptoms of Sjögren's syndrome (SS). Xerostomia is the subjective sensation of dry mouth, which is often, but not always, associated with hypofunction of the salivary glands. In 2016, it was included among the preliminary SS diagnostic criteria [1]. The unstimulated salivary flow (UWS) test is one of the objective SS classification criteria used to assess the severity of salivary glands' hypofunction. A salivary flow rate of $\leq 0.1 \mathrm{~mL} / \mathrm{min}$ is considered an abnormal result typical for SS. This value was first specified in the classification criteria established more than twenty years ago [2] and has not been changed to date. Recently, an increasing number of studies is being published in which the authors prove that UWS values are age- and gender-dependent [3, 4]. Unfortunately, 
in the majority of the cases, this test is only applied when the patient does not have a dry eye by objective measures [1].

Labial salivary gland biopsy (LSGB) is considered one of the most objective tests used to confirm the diagnosis of SS [1, 2]. Despite its diagnostic value, LSGB [5] has certain limitations [6] manifested, e.g., by false-positive results in older adults. Hence, the question naturally arises as to which patients should be subjected to LSGB with a high likelihood of SS diagnosis.

The study's objective was to evaluate UWS rate measurements in screening patients with suspected Sjögeren syndrome and presenting with xerostomia in whom LSGB should be performed. We analyze the correlation between UWS value and focus score (FS) and anti-SSA antibodies. We will try to answer whether it is possible not to perform LSGB in targeted patients according to UWS results $>0.1 \mathrm{~mL} / \mathrm{min}$ ? Is there a UWS value at which we can determine with high probability that we will get a focus score above 1 ?

\section{Materials and methods}

The study group consisted of subjects above 18 years of age who had reported to the Rheumatology Clinic with suspected SS and subjective feeling of oral dryness between December 2018 and September 2020. Each subject had provided at least one positive response in the oral dryness symptoms inventory per the AECG/ACR criteria $[1,2]$ as inclusion criteria. Each person had provided written consent to undergo study procedures including UWS measurements [7], LSGB, collection of blood samples with the determination of ANA (IF), ENA (ELISA), anti-dsDNA (ELISA) antibodies, ultrasound (US) of large salivary glands (the MyLab25Gold (Esaote) with a linear head; frequency range: $12-18 \mathrm{MHz}$ ), and Schirmer's test. The US examination was blinded for laboratory parameters. The diagnosis of SS was confirmed based on the ACR/EULAR 2016 classification criteria [1]. Exclusion criteria included: age $<18$ years, diagnosis of other autoimmune diseases, active HCV infection, sarcoidosis, IgG4-related disease, lymphoma, amyloidosis, graft-versus-host disease.

The control group consisted of patients with xerostomia with suspected pSS, who were not confirmed as having pSS or other autoimmune diseases.

The prospective-observational study was approved by the Ethics Committee of Medical University (decision number 599/2018) and carried out according to the Declaration of Helsinki.

UWS tests were carried out in the morning (8:00 am-12:00 pm). Each subject had been asked not to use any parasympathomimetic drugs during the preceding $12 \mathrm{~h}$, not to smoke or drink coffee, not to use any drugs $3 \mathrm{~h}$ before the test, not to use any saliva substitutes $3 \mathrm{~h}$ before the test, as well as not to eat, drink water, and brush or floss their teeth one hour before the test.

After the test, LSGB was collected from each subject, following topical mucosal anesthesia using $10 \%$ lidocaine, for histopathological screening for lymphocytic infiltrations typical for SS as per current guidelines (focal lymphocytic sialadenitis and focus score (FS) of $\geq 1$ foci/ $4 \mathrm{~mm}^{2}$ ) [8]. All subjects were then subjected to the ultrasound of major salivary glands to identify hypoechogenic areas and blurred and patchy salivary gland structures typical of SS as defined in the classification system proposed by De Vita [9].

\section{Statistical analysis}

At the first stage, homogeneity between pSS and the control group was analyzed. At the second stage, patients with pSS were divided into subgroups of patients presenting with SSA antibodies (SS-A + ) and patients without SSA antibodies (SS-A -). Differences between the groups were evaluated.

Statistical analyses were carried out using the Statistica 10 software package.

Mann-Whitney $U$ test was used to compare the distributions of quantitative variables in two independent groups. A chi-squared test was used to verify the relationships between variables expressed using dichotomous scales. Spearman's coefficient and the corresponding statistical test were used to assess the significance of correlations between quantitative variables. The significance level was set to 0.05 .

\section{Results}

A total of 105 subjects with subjective sensation on the dry mouth (xerostomia) were qualified for the study. The final diagnosis of pSS was made in 44 patients. In 61 people with subjective xerostomia, the pSS or other defined disease was not diagnosed (control group) (Table 1).

No age differences were identified between pSS and control group subjects. The majority of patients were females around 55 years of age. In the first stage, we analyzed the UWS values' dependence on patients' age (Fig. 1). Statistical analysis showed that UWS values were not significantly lower in older individuals with pSS. The calculated Spearman's correlation coefficient was -0.28 . The $p$ value for the correlation significance test was -0.23 .

The Schirmer test $(<5 \mathrm{~mm}$ in $5 \mathrm{~min}$ at least in one eye) was positive in $86 \%(n=38)$ of patients with pSS. The dry eye in Schirmer test (result $<15 \mathrm{~mm}$ in $5 \mathrm{~min}$ at least in one eye) was confirmed in $49 \%$ of patients $(n=30)$ from the control group, but only in $8 \%(n=5)$ the Schirmer test was lower than $5 \mathrm{~mm}$ in $5 \mathrm{~min}$. 
Table 1 Characteristics of the pSS patients and the control group

Fig. 1 Correlation between unstimulated whole salivary flow (UWS) rate and age of patients in group with primary Sjögren syndrome
pSS ( $n$ 44)

Control group ( $n$ 61) with subjec- $p$ value tive xerostomia

\begin{tabular}{|c|c|c|c|}
\hline \multicolumn{4}{|l|}{ Patient age (years) } \\
\hline Mean (SD) & $55(14.6)$ & $55(11.6)$ & \\
\hline Range & $26-81$ & $26-75$ & \\
\hline \multicolumn{4}{|l|}{ Sex } \\
\hline Females $(n)$ & 39 & 57 & \\
\hline Males $(n)$ & 5 & 4 & \\
\hline Mean USF (mL/min) & 0.33 & 0.67 & .003 (test $U$ ) \\
\hline \multicolumn{4}{|l|}{$\mathrm{UWS} \leq 0.1 \mathrm{~mL} / \mathrm{min}$} \\
\hline$n$ & 15 & 6 & \multirow[t]{2}{*}{.01 (test $\left.\chi^{2}\right)$} \\
\hline$\%$ & 34 & 10 & \\
\hline Mean FS (LSGB) & 2.0 & 0.05 & $<.005($ test $U)$ \\
\hline \multicolumn{4}{|l|}{$\mathrm{FS} \geq 1(\mathrm{LSGB})$} \\
\hline$n$ & 25 & 1 & \multirow{2}{*}{$<.05\left(\right.$ test $\left.\chi^{2}\right)$} \\
\hline$\%$ & 57 & 1 & \\
\hline \multicolumn{4}{|c|}{$\begin{array}{l}\text { Changes in salivary gland US } \\
\text { scans }\end{array}$} \\
\hline \multicolumn{4}{|l|}{ Parotid } \\
\hline$n$ & 28 & 5 & \multirow[t]{2}{*}{$<.005$ (test $\left.\chi^{2}\right)$} \\
\hline$\%$ & 63 & 8 & \\
\hline \multicolumn{4}{|l|}{ Submandibular } \\
\hline$n$ & 31 & 7 & \multirow[t]{2}{*}{$<.005$ (test $\left.\chi^{2}\right)$} \\
\hline$\%$ & 70 & 11 & \\
\hline \multicolumn{4}{|l|}{$\mathrm{ANA} \geq 1: 320$} \\
\hline$n$ & 39 & 19 & \multirow[t]{2}{*}{$.002\left(\right.$ test $\left.\chi^{2}\right)$} \\
\hline$\%$ & 89 & 31 & \\
\hline
\end{tabular}

ANA antinuclear antibodies; FS focus score; UWS unstimulated salivary flow; US scans ultrasonography based on de Vita score; $L S G B$ labial salivary gland biopsy; $n$ number of patients

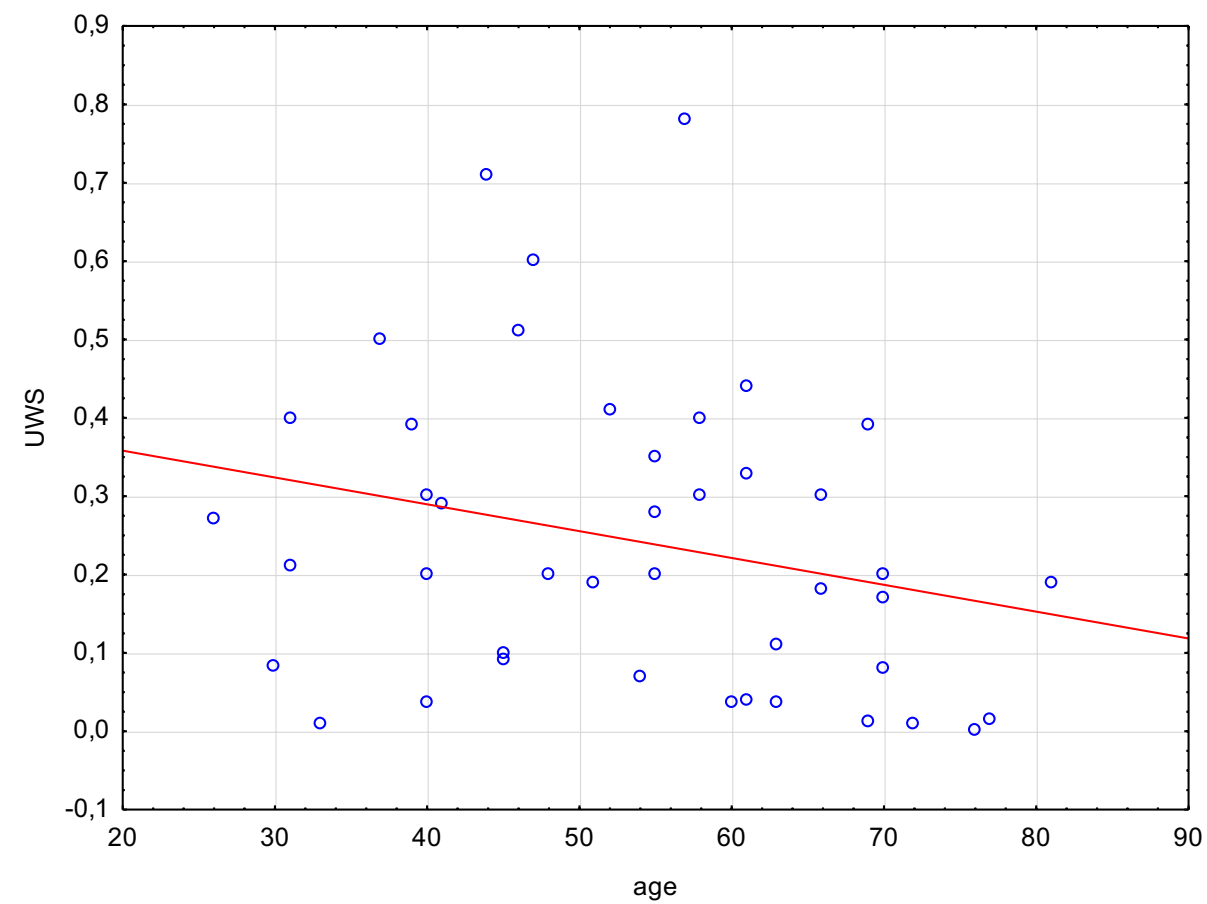


UWS rates were significantly lower in pSS patients compared to the control group of subjects with subjective xerostomia $(0.33 \mathrm{~mL} / \mathrm{min}$ vs $0.67 \mathrm{~mL} / \mathrm{min}, p<0.05)$. Likewise, lymphocytic infiltrations of salivary glands were significantly more common in pSS patients $(57 \%$ vs $1 \%, p<005)$. However, no association was identified between UWS and FS $\geq 1$ (Fig. 2). As much as $65 \%$ of pSS patients presented with UWS of $>0.1 \mathrm{~mL} / \mathrm{min}$.

Changes in ultrasound scans of large salivary glands consisting of structural heterogeneity and hypoechogenic areas were observed more frequently in pSS patients than control subjects. Within the pSS group, changes were more frequent in submandibular salivary glands than parotid salivary glands (70\% vs $63 \%)$, albeit the difference was not statistically significant $(p>0.05)$. Changes in ultrasound scans of large salivary glands were observed in $11 \%$ of control group subjects, mainly within the submandibular salivary glands. ANA at titers of $\geq 1: 320$ was confirmed in $31 \%$ of control group subjects. However, no criteria of SS or other systemic connective tissue disease were met by these subjects. Most frequently, antibodies observed in this group included anti-DFS70 (80\%), AMA, anti-centromere and anti-Ro52 antibodies.

No differences were observed between anti-SSA + and anti-SSA - patients in terms of age, UWS rates, and presence of ultrasound imaging changes (Table 2).

\section{Discussion}

Our study results indicate that UWS rates are significantly lower in the group of patients with confirmed pSS compared to those with subjective xerostomia without autoimmune background. However, only less than one-half of the measurements are below the value of $0.1 \mathrm{~mL} / \mathrm{min}$ adopted as the threshold in the classification criteria at the time of diagnosis. In our study population, changes in ultrasound imaging of salivary glands occurred almost exclusively among subjects who met the SS diagnostic criteria. Another important observation was that SSA + and SSA - patients did not differ in terms of age, UWS rates, or changes in large salivary gland ultrasound scans. Therefore, LSGB should be a useful and routine component of diagnostic examinations.

SS is most likely to be suspected in patients showing signs of eye and mouth dryness. UWS rate measurements are a simple, non-invasive, and repeatable procedure providing an objective method for quantitative assessment of salivary secretion. When assessing UWS rates, possible factors responsible for xerostomia should be taken into account and eliminated already at the preliminary study preparation stage $[10,11]$. When assessing the results, the patient's age and gender should also be considered since UWS rates are lower in females above $50[4,12]$. The same holds for male patients [4, 13]. However, antihypertensive treatment was found to have no impact on UWS rates in pSS patients [4]. Despite being considered one of the major causes of decreased UWS values (an iatrogenic
Fig. 2 Relationship between focus score $\geq 1$ (FS) and unstimulated whole salivary flow (UWS) values in patients with primary Sjögren's syndrome

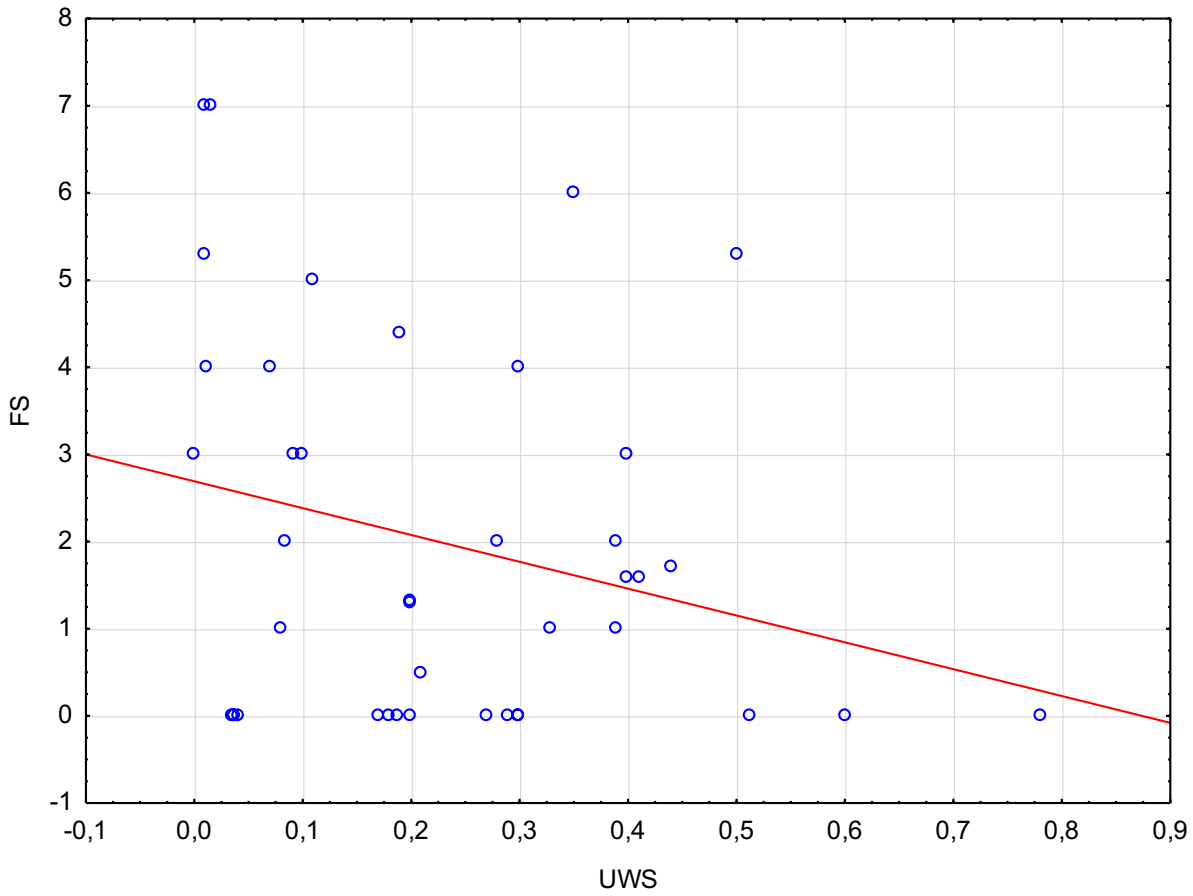


Table 2 Characteristics of pSS patients with and without antiSSA antibodies

\begin{tabular}{|c|c|c|c|}
\hline & $\begin{array}{l}\text { pSS-positive } \\
\text { anti SSA ab ( } n \text { 34) }\end{array}$ & $\begin{array}{l}\text { pSS-negative } \\
\text { anti SSA ab ( } n 10)\end{array}$ & $p$ value \\
\hline \multicolumn{4}{|l|}{ Patient age (years) } \\
\hline Mean (SD) & $53(14.5)$ & $60(14.0)$ & .12 (test $U$ ) \\
\hline Mean UWS (mL/min) & 0.4 & 0.09 & .60 (test $U$ ) \\
\hline \multicolumn{4}{|l|}{$\mathrm{UWS} \leq 0.1 \mathrm{~mL} / \mathrm{min}$} \\
\hline $\begin{array}{l}n \\
\%\end{array}$ & $\begin{array}{l}11 \\
32\end{array}$ & $\begin{array}{l}4 \\
40\end{array}$ & .75 (test $\left.\chi^{2}\right)$ \\
\hline Mean FS (LSGB) & 1.7 & 2.9 & .015 (test $U$ ) \\
\hline \multicolumn{4}{|l|}{$\mathrm{FS} \geq 1(\mathrm{LSGB})$} \\
\hline $\begin{array}{l}n \\
\%\end{array}$ & $\begin{array}{l}26 \\
76\end{array}$ & $\begin{array}{l}10 \\
100\end{array}$ & .60 (test $\chi^{2}$ ) \\
\hline \multicolumn{4}{|c|}{$\begin{array}{l}\text { Changes in salivary gland US } \\
\text { scans }\end{array}$} \\
\hline \multicolumn{4}{|l|}{ Parotid } \\
\hline$n$ & 25 & 4 & \multirow[t]{2}{*}{.34 (test $\left.\chi^{2}\right)$} \\
\hline$\%$ & 73 & 40 & \\
\hline \multicolumn{4}{|l|}{ Submandibular } \\
\hline$n$ & 25 & 6 & \multirow[t]{2}{*}{.72 (test $\left.\chi^{2}\right)$} \\
\hline$\%$ & 73 & 60 & \\
\hline
\end{tabular}

ANA antinuclear antibodies; FS focus score; UWS unstimulated salivary flow; US scans ultrasonography based on de Vita score; $L S G B$ labial salivary gland biopsy; $n$ number of patients; $a b$ antibodies factor). In our analysis, we have not confirmed the relationship between the value of UWS and age. UWS values were lower in individuals over 50. The calculated Spearman's correlation coefficient was -0.28 . The $p$ value for the correlation significance test was 0.1 , which means that correlation exists, albeit it is very weak and not significant at the predefined significance level of $5 \%$. Nonetheless, a correlation exists and must be examined in a larger population.

Authors of a Scandinavian study claim that lower UWS rates are negatively correlated with the immune parameters such as ANA, anti-SSA, and anti-SSB antibodies; No association between the UWS rate and the FS, patient's age, and duration of pSS could be demonstrated [14], as also confirmed by our results. We did not observe the correlation between UWS and anti-SSA positivity.

Recent studies suggested the need to revalidate UWS threshold values in the diagnostics of pSS depending on the factors mentioned above. The UWS threshold of $0.1 \mathrm{~mL} /$ min was proposed in 1992 when pSS classification criteria were established [2] and have not been verified ever since. Lacombe et al. [4] also observed the same results as we, that pSS patients presented higher mean UWS rates $(0.3 \mathrm{~mL} /$ min in our study). In our group, as many as $65 \%$ of $\mathrm{pSS}$ patients had UWS of $>0.1 \mathrm{~mL} / \mathrm{min}$, indicating the need for a thorough evaluation of the previous UWS threshold as used in the classification criteria. Researchers of a recently published study came to similar conclusions. In a meta-analysis on UWS in SS, they suggest the need for further study to redefine UWS cut-off points to better discriminate between patients with SS vs. sicca non-SS [15].

Although LSGB is an invasive procedure, it is quite simple in technical terms and does not require significant financial resources, including anesthesiological preparation and full operating block conditions. The procedure can be performed after the patient has been prepared in the treatment room. From our experience, LSGB following topical anesthetic spray application is well-tolerated by patients and very rarely involves local complications. Patients presenting with anti-SSA antibodies were not different from antibodynegative patients in terms of age, UWS rate, or changes in major salivary gland ultrasound scans.

In addition, large-scale analyses of $\mathrm{pSS}$ patients revealed an association between the presence of lymphocyte infiltrations meeting the FS criteria and anti-SSA and anti-SSB antibodies, RF, higher IgG levels, and clinical signs of dryness of both mouth and eyes [16, 17].

Besides, higher FS values were associated with the possibility of lymphomas developing in the course of SS [18]. Hence, thorough histopathological evaluation of LSGB specimens, including germinal centers' assessment, may be of prognostic importance as early as at the SS diagnostic stage [19]. Given the above data, this is another reason to assess FS regardless of the value of the UWS.

The logistic regression analysis results revealed that severe US activity was an independent predictor of moderate and high pSS activity [20]. The presence of lymphoma increased significantly, with a higher risk for moderate 
disease activity. It is all the more indicative of the need to perform LSGBs to evaluate SS activity, particularly in patients with xerostomia and UWS of $<0.4 \mathrm{~mL} / \mathrm{min}$. In our patients, changes observable in ultrasound scans of major salivary glands were found mainly in pSS patients. They were nearly completely lacking in the control group. Given this, we believe that along with the UWS rate measurements, changes observable in ultrasound scans of major salivary glands may comprise a significant non-invasive criterion in SS diagnostics.

The limitations of this study include a small sample of the study group, which needs further studies.

In conclusion, LSGB should be performed in all suspected pSS cases, regardless of the UWS rate value. In patients with suspected pSS, only less than one-half of the UWS measurements are below the value of $0.1 \mathrm{~mL} / \mathrm{min}$ adopted as the threshold in the classification criteria for pSS.

Acknowledgements We would like to thank Marek Frankowski, Małgorzata Chowaniec, and Marek Bubnowski for their help in referring patients to diagnostics.

Author contribution AS, MM, and MS conceived the concept of the study, contributed to the design of the research. AS, MM, MS, KP, and $\mathrm{KZ}$ authors were involved in data collection. AS, MM, MS, PW, KCW, and PW analyzed the data. All authors edited and approved the final version of the manuscript.

Funding None.

\section{Declarations}

Conflict of interest The authors declare that there is no conflict of interest.

Open Access This article is licensed under a Creative Commons Attribution 4.0 International License, which permits use, sharing, adaptation, distribution and reproduction in any medium or format, as long as you give appropriate credit to the original author(s) and the source, provide a link to the Creative Commons licence, and indicate if changes were made. The images or other third party material in this article are included in the article's Creative Commons licence, unless indicated otherwise in a credit line to the material. If material is not included in the article's Creative Commons licence and your intended use is not permitted by statutory regulation or exceeds the permitted use, you will need to obtain permission directly from the copyright holder. To view a copy of this licence, visit http://creativecommons.org/licenses/by/4.0/.

\section{References}

1. Shiboski CH, Shiboski SC, Seror R et al (2017) 2016 American College of Rheumatology/European League Against Rheumatism Classification Criteria for Primary Sjögren's Syndrome: a consensus and data-driven methodology involving three international patient cohorts. ArthritisRheumatol 69:35-45. https://doi.org/10. 1002/art.39859

2. Vitali C, Bombardieri S, Moutsopoulos HM et al (1993) Preliminary criteria for the classification of Sjögren's syndrome. Results of a prospective concerted action supported by the European community. ArthritisRheum 36:340-347. https://doi.org/10.1002/art. 1780360309

3. Affoo RH, Foley N, Garrick R et al (2015) Meta-analysis of salivary flow rates in young and older adults. J Am Geriatr Soc 63(10):2142-2151. https://doi.org/10.1111/jgs.13652

4. Lacombe V, Lacout C, Lozac'h P et al (2020) Unstimulated whole saliva flow for diagnosis of primary Sjögren'ssyndrome: time to revisit the threshold? Arthritis Res Ther 22(1):38. https://doi.org/10.1186/s13075-020-2132-3

5. Sebastian A, Szachowicz A, Wiland P (2019) Classification criteria for secondary Sjögren's syndrome. Current state of knowledge. Reumatologia 57(5):277-280. https://doi.org/10.5114/reum.2019. 89520

6. Manzo C (2019) Labial salivary gland biopsy and secondary Sjögren's syndrome: where we are and where we want to be. Reumatologia 57(6):354-355. https://doi.org/10.5114/reum.2019. 91274

7. Navazesh M, Kumar SK (2008) University of Southern California School of Dentistry. Measuring salivary flow: challenges and opportunities. J Am Dent Assoc 139:35S-40S. https://doi.org/10. 14219/jada.archive.2008.0353

8. Fisher BA, Jonsson R, Daniels T et al (2017) Standardisation of labial salivary gland histopathology in clinical trials in primary Sjögren's syndrome. Ann Rheum Dis. 76(7):1161-1168. https:// doi.org/10.1136/annrheumdis-2016-210448

9. Qi X, Sun C, Tian Y et al (2017) Comparison of the diagnostic value of four scoring systems in primary sjögren's syndrome patients. Immunol Lett 188:9-12. https://doi.org/10.1016/j.imlet. 2017.05.009

10. Baer AN, Walitt B (2017) Sjögren syndrome and other causes of sicca in older adults. ClinGeriatr Med 33:87-103. https://doi.org/ 10.1016/j.cger.2016.08.007

11. Wolff A, Joshi RK, Ekström J et al (2017) A guide to medications inducing salivary gland dysfunction, xerostomia, and subjective sialorrhea: a systematic review sponsored by the world workshop on oral medicine VI. Drugs RD 17:1-28. https://doi.org/10.1007/ s40268-016-0153-9

12. Speight PM, Kaul A, Melsom RD (1992) Measurement of whole unstimulated salivary flow in the diagnosis of Sjögren's syndrome. Ann Rheum Dis 51:499-502. https://doi.org/10.1136/ard.51.4.499

13. Affoo RH, Foley N, Garrick R et al (2015) Meta-analysis of salivary flow rates in young and older adults. J Am GeriatrSoc 63:2142-2151. https://doi.org/10.1111/jgs. 13652

14. Haga HJ (2002) Clinical and immunological factors associated with low lacrimal and salivary flow rate in patients with primary Sjögren's syndrome. J Rheumatol. 29(2):305-308

15. Martínez-Ceballos MA, Aguilera N, Garzón-González KC et al (2021) Unstimulated whole salivary flow in Sjögren's Syndrome: systematic literature review and meta-analysis. Adv Rheumatol. 61(1):8. https://doi.org/10.1186/s42358-020-00158-0

16. Daniels TE, Whitcher JP (1994) Association of patterns of labial salivary gland inflammation with keratoconjunctivitis sicca. Analysis of 618 patients with suspected Sjogren's syndrome. Arthritis Rheum 37:869-877. https://doi.org/10.1002/art.1780370615

17. Daniels TE, Cox D, Shiboski CH et al (2011) Sjogren's International Collaborative Clinical Alliance Research Associations between salivary gland histopathologic diagnoses and phenotypic features of Sjogren's syndrome among 1,726 registry participants. Arthritis Rheum 63:2021-2030

18. Carubbi F, Alunno A, Cipriani P et al (2015) A retrospective, multicentre study evaluating the prognostic value of minor salivary gland histology in a large cohort of patients with primary Sjögren's syndrome. Lupus 24(3):315-320. https://doi.org/10. $1177 / 0961203314554251$ 
19. Barone F, Campos J, Bowman S, Fisher BA (2015) The value of histopathological examination of salivary gland biopsies in diagnosis, prognosis and treatment of Sjögren's Syndrome. Swiss Med Wkly 16(145):w14168. https://doi.org/10.4414/smw.2015.14168

20. Milic V, Colic J, Cirkovic A et al (2019) Disease activity and damage in patients with primary Sjogren's syndrome: prognostic value of salivary gland ultrasonography. PLoS ONE 14:e0226498. https://doi.org/10.1371/journal.pone.0226498
Publisher's Note Springer Nature remains neutral with regard to jurisdictional claims in published maps and institutional affiliations. 